\title{
Fibrinogen/albumin ratio as a promising predictor of platinum response and survival in ovarian clear cell carcinoma
}

Wei Chen ${ }^{1,2+}$, Boer Shan ${ }^{1,3+}$, Shuling Zhou ${ }^{3,4}$, Huijuan Yang ${ }^{1,3^{*}}$ and Shuang Ye $\mathrm{e}^{1,3^{*}}$

\begin{abstract}
Background: This study aims to evaluate the role of the fibrinogen/albumin ratio (FAR) in predicting platinum resistance and survival outcomes of patients with ovarian clear cell carcinoma (OCCC).

Methods: Coagulation function and D-dimer, serum albumin, CA125 and HE4 levels were measured before surgery in OCCC patients undergoing initial surgery in our institution. FAR was calculated as fibrinogen/albumin level. The correlation between these indicators and clinicopathological features, platinum response, and survival outcomes was further analyzed. The Kaplan-Meier method and multivariable Cox regression model were used to assess the effects of FAR on progression-free survival (PFS) and overall survival (OS).

Results: Advanced stage patients accounted for $42.1 \%$ of the 114 participants. Optimal cytoreductive surgery was achieved in 105 patients, and the complete resection rate was 78.1\%. FAR was associated with tumor stage, residual tumor and platinum response. A receiver operating characteristic curve for predicting platinum response showed that the optimal cutoff point of the FAR was $12 \%$. The sensitivity was $73.3 \%$ and the specificity was $68.2 \%$. In multivariate analysis, $F A R \geq 12 \%(H R=4.963, P=0.002)$ was an independent risk factor for platinum resistance. In addition, FAR and D-dimer proved to be independent negative factors for outcomes including both PFS and OS. The median follow-up time was 52 months. A high FAR ( $\geq 12 \%$ ) showed a stronger correlation with poor OS and PFS in the subgroup analysis of advanced and completely resected patients.
\end{abstract}

Conclusions: The FAR might be a potential preoperative biochemical marker for predicting treatment response and oncological outcomes in OCCC patients.

Keywords: Ovarian neoplasms, Clear cell carcinoma, Fibrinogen/albumin ratio, Platinum resistance, Survival

\section{Background}

Ovarian cancer is one of the most lethal diseases among gynecological malignancies [1]. Seventy percent of ovarian cancers are epithelial, of which ovarian clear cell carcinoma (OCCC) is a specific type of epithelial ovarian cancer (EOC) with a higher incidence in East Asian

\footnotetext{
*Correspondence: huijuanyang@hotmail.com; mendy_ye@126.com

${ }^{\dagger}$ Wei Chen and Boer Shan contributed equally to this work.

${ }^{3}$ Department of Oncology, Shanghai Medical College, Fudan University,

Shanghai 200032, China

Full list of author information is available at the end of the article
}

women [2]. Tumor stage, residual tumor and chemotherapy response are recognized as prognostic factors for survival in ovarian cancer [3]. Although many patients with OCCC are diagnosed at an early stage, their prognosis is still worse due to disease aggressiveness and platinum resistance compared to their serous counterparts, which account for the highest proportion $[4,5]$. In addition, OCCC patients who harbor the same stage or residual tumors also show obvious heterogeneous survival due to their unpredictable response to platinum [6]. The high cost of genetic markers limits their application in clinical 
practice. Therefore, a simple, economical and effective biomarker that accurately reflects the platinum response and survival outcome of the disease is needed.

Hypercoagulability is associated with malignancy and is more pronounced in patients with OCCC [7]. For example, patients with OCCC tend to show higher D-dimer than other patients with EOC [8]. In addition, fibrinogen, one of the important indicators of the coagulation function, is also an acute-phase protein, and its plasma level increases during the systemic inflammatory response [9]. It has attracted increasing attention because of its important role in the development and progression of inflammation and cancer [10-12]. Many studies on malignant tumors have shown that elevated plasma fibrinogen levels before surgery are significantly associated with treatment failure or adverse outcomes in patients [11]. Furthermore, the fibrinogen/albumin ratio (FAR) is considered to be an important biomarker reflecting the systemic inflammatory state and nutritional state. It has been reported that FAR is closely related to the prognosis of various cancers $[9,13-17]$. However, few studies have explored the value of the FAR in OCCC.

Therefore, we tried to evaluate the role of the FAR as a predictive and prognostic biochemical marker in OCCC in a well-annotated cohort involving 114 patients treated in our institution.

\section{Materials and methods \\ Patients}

This retrospective study was approved by the Ethics Committee of Fudan University Shanghai Cancer Center, and the written informed consent requirement was waived due to its retrospective design. We searched our electronic medical record systems for all OCCC patients who underwent their initial surgery at our institution between 2007 and 2018. The inclusion criteria were as follows: 1 ) pathological diagnosis of OCCC; 2 ) no preoperative neoadjuvant chemotherapy; 3 ) no other malignant tumor; and 4) no signs of venous thromboembolism, including deep vein thrombosis and pulmonary embolism, at first diagnosis.

The interval between preoperative blood tests and surgery is usually less than seven days. Coagulation function tests (including fibrinogen), plasma D-dimer, serum albumin, HE4 and CA125 in routine preoperative examinations were retrospectively extracted from medical records. FAR is defined as the fibrinogen/albumin ratio. Comprehensive staging surgery was performed for the early-stage patients (stage I+II), and debulking reduction was performed for the advanced patients (stage III +IV). Optimal cytoreductive surgery means that the size of the residual tumor is less than or equal to $1 \mathrm{~cm}$. Complete resection means no visible tumor after cytoreduction. Both recurrence and progression after treatment are based on imaging evidence (CT or MRI). Platinum resistance was defined as those who completed standard platinum-based chemotherapy and progressed within six months after the last chemotherapy session. Progression-free survival (PFS) and overall survival (OS) were defined as the time interval between the date of the primary surgery to the date of first recurrence and death/ last contact, respectively.

In our institution, patients with venous thromboembolism (VTE) at first diagnosis usually receive anticoagulation (low molecular weight heparin during the study period) and neoadjuvant chemotherapy. Therefore, those patients were not included in the study considering that VTE, anticoagulation treatment and neoadjuvant chemotherapy might all affect the serum coagulation panels to different extents. During the study period, ovarian cancer patients usually receive platinum-based chemotherapy (paclitaxel $175 \mathrm{mg} / \mathrm{m} 2$ and carboplatin $\mathrm{AUC}=5$ ) after surgery. Patients with advanced tumors are required to receive at least six cycles. For those with early-stage disease, the number of cycles ranged from four to six after being tailored to different individuals. Postoperative radiation and maintenance treatment were not recommended.

\section{Statistical analysis}

Clinical variables were described by descriptive statistics. Median and range were used for continuous variables, and proportions were used for classified data. Receiver operating characteristic (ROC) curves was established, and the optimal cutoff threshold of the FAR for predicting platinum response, PFS and OS were determined by Youden's index correction. The baseline features compared using the Mann-Whitney $\mathrm{U}$ test due to nonnormal distribution. Univariate and multivariate analyses were performed based on the log-rank test and Cox regression, respectively. Survival time was estimated by the KaplanMeier model. All reported $P$ values were double-tailed, and $P<0.05$ was considered statistically significant. Statistical analyses were performed with SPSS Version 24.0 (SPSS, Inc., Chicago, IL, USA). ROC curves and KaplanMeier curves were plotted using GraphPad Prism 6.0 (GraphPad Software, Inc., La Jolla, CA).

\section{Result}

\section{Clinical characteristics of the study population} and the association between FAR and platinum response A total of 120 OCCC patients met the eligibility criteria, and six patients were excluded for incomplete clinical data. Of these, 66 patients (57.9\%) with early-stage disease (I+II) received staging surgery, and 48 patients (42.1\%) with advanced-stage disease (III + IV) underwent 
debulking surgery. Optimal cytoreductive surgery was achieved in 105 patients. The flow chart of study participant inclusion and exclusion is shown in Fig. 1.

Table 1 shows the median and range of preoperative biochemical variables (CA125, HE4, albumin, fibrinogen, D-dimer) stratified by the clinicopathological features of the 114 patients. The values of these indicators are significantly different in patients with platinum sensitivity and platinum resistance. FAR was associated with endometriosis, stage, residual tumor and platinum response. The distribution and proportion of FAR (\%) are shown in Fig. 2.
The ROC curve predicting the outcome of platinumbased chemotherapy was generated, of which the area under the curve (Fig. 3) was 0.736 to verify that the optimal cutoff point FAR was $12 \%$. The sensitivity was $73.3 \%$, and the specificity was $68.2 \%$. Among 114 patients included in the analysis, 63 patients had FAR $<12 \%$, and 51 patients had FAR $\geq 12 \%$. The relationships between preoperative peripheral blood variables and platinum response are presented in Table 2. On univariate analysis, variables such as FIGO stage (hazard ratio $(\mathrm{HR})=2.526$, $P<0.001$ ), albumin (HR $=0.903, P=0.055)$, fibrinogen $(\mathrm{HR}=1.516, P=0.016)$, D-dimer $(\mathrm{HR}=1.116, P=0.027)$,

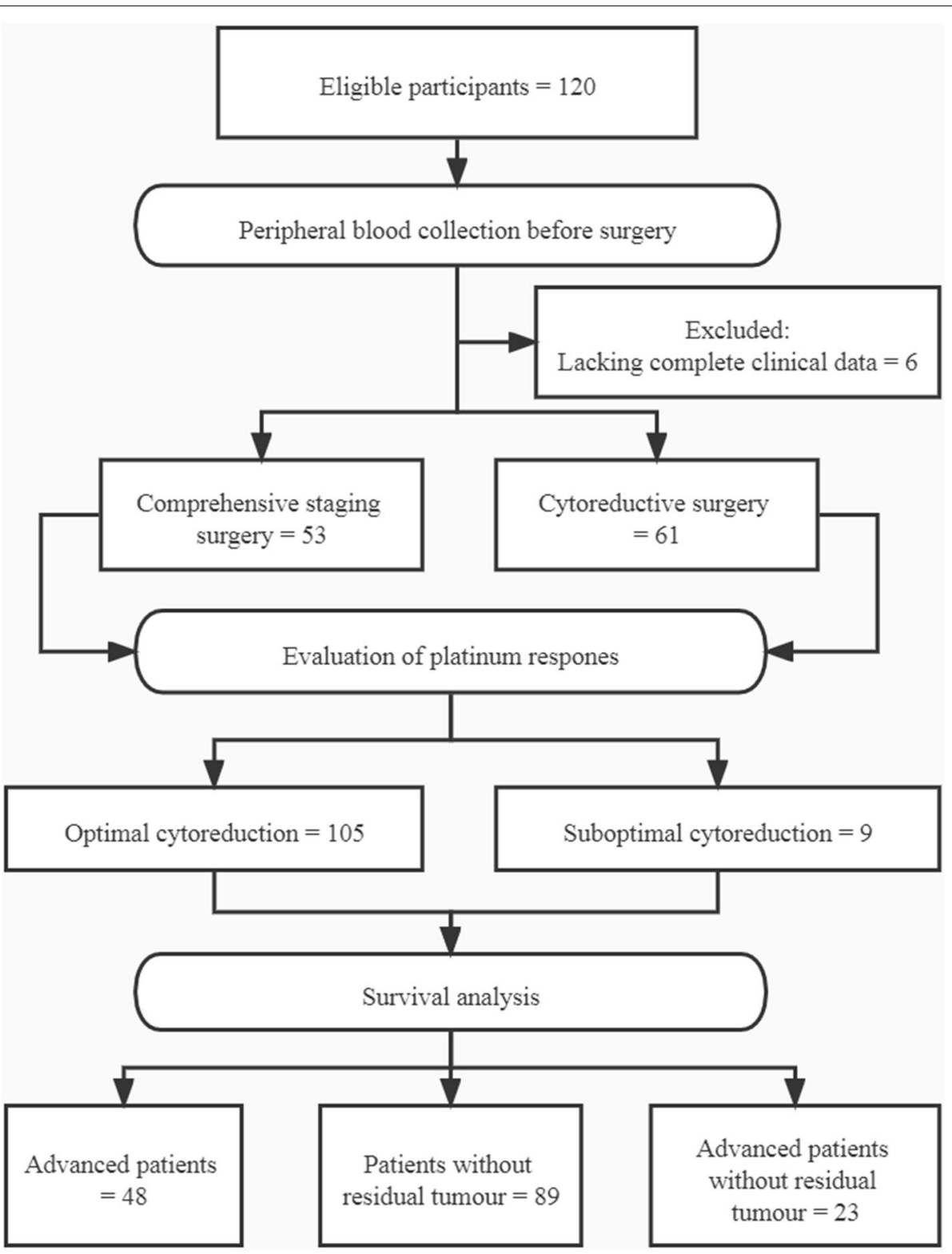

Fig. 1 Flow chart illustrating the patient inclusion 
Table 1 Patient characteristics in relation to preoperative blood parameters

\begin{tabular}{|c|c|c|c|c|c|c|c|}
\hline Characteristic & $\mathrm{N}^{\mathrm{a}}(\%)$ & $\mathrm{CA} 125^{\mathrm{b}}(\mathrm{U} / \mathrm{ml})$ & $\begin{array}{l}\mathrm{HE}^{\mathrm{b}}{ }^{-} \\
(\mathrm{pmol} / \mathrm{L})\end{array}$ & $\begin{array}{l}A^{A L B}{ }^{b} \\
(g / l)\end{array}$ & $\mathrm{FIB}^{\mathrm{b}}(\mathrm{g} / \mathrm{l})$ & FAR $^{b}(\%)$ & $\begin{array}{l}\mathrm{DDI}^{\mathrm{b}} \\
(\mathrm{mg} / \mathrm{l})\end{array}$ \\
\hline All cases & 114 & $186.1\left(6.5-5000^{*}\right)$ & $106.2\left(37.0-1500^{*}\right)$ & $41.1(25.9-52.1)$ & $4.58(1.94-8.94)$ & $10.7(4.8-25.1)$ & $1.5(0.1-55.2)$ \\
\hline \multicolumn{8}{|l|}{ Age (year) } \\
\hline$<40$ & $12(10.5)$ & $254.7(66.2-912.8)$ & 111.1 (35.5-209.6) & $42.2(33.7-49.2)$ & $4.85(2.69-6.59)$ & $11.4(6.5-16.3)$ & $2.1(0.7-10.7)$ \\
\hline $40 \sim 59$ & $75(65.8)$ & $131.0(6.5-2854.0)$ & $106.2\left(37.0-1500^{*}\right)$ & $41.5(28.1-52.1)$ & $4.34(1.94-8.94)$ & $10.2(4.9-25.1)$ & $1.4(0.1-55.2)$ \\
\hline$\geq 60$ & $27(23.7)$ & $239.1\left(13.29-5000^{*}\right)$ & $124.3\left(55.5-1500^{*}\right)$ & $40.3(25.9-46.5)$ & $4.38(2.42-8.79)$ & $12.1(6.2-23.3)$ & $2.3(0.2-30.6)$ \\
\hline$P$ value & & 0.081 & 0.228 & 0.377 & 0.778 & 0.074 & 0.451 \\
\hline \multicolumn{8}{|l|}{ FIGO stage } \\
\hline 1 & $53(46.5)$ & $66.2\left(6.5-5000^{*}\right)$ & $106.2\left(37.0-1500^{*}\right)$ & $42.1(28.1-52.1)$ & $3.90(1.94-7.15)$ & $8.9(4.9-21.6)$ & $0.7(0.1-42.6)$ \\
\hline$\|$ & $13(11.4)$ & 133.5 (22.2-1401.0) & $106.2(47.0-252.4)$ & $43.7(31.5-47.8)$ & $4.04(2.30-7.92)$ & $9.1(5.2-25.1)$ & $1.3(0.3-55.2)$ \\
\hline III & $35(30.7)$ & $283.8(46.1-2854.0)$ & $106.2\left(47.4-1500^{*}\right)$ & $40.3(30.8-46.5)$ & $4.83(2.20-8.94)$ & $12.0(5.4-23.3)$ & $3.2(0.5-11.0)$ \\
\hline IV & $13(11.4)$ & $304.8\left(48.9-5000^{*}\right)$ & $106.2(44.1-331.9)$ & $39.2(25.9-49.2)$ & $4.75(3.42-7.95)$ & $15.1(8.8-11.5)$ & $3.9(0.6-9.2)$ \\
\hline$P$ value & & 0.006 & 0.837 & 0.018 & 0.180 & 0.007 & 0.008 \\
\hline \multicolumn{8}{|l|}{ Residual tumour } \\
\hline 0 & $89(78.1)$ & $126.0\left(6.5-5000^{*}\right)$ & $106.2(37.0-1500 *)$ & $41.7(28.1-52.1)$ & $4.13(1.94-8.94)$ & $9.9(4.9-25.1)$ & $1.2(0.1-55.2)$ \\
\hline$\leq 1 \mathrm{~cm}$ & $16(14.0)$ & $551.9(46.1-1845.3)$ & $112.7(68.9-904.0)$ & $39.0(29.0-45.4)$ & $4.98(3.25-8.79)$ & $12.9(7.3-15.9)$ & $3.9(0.7-9.3)$ \\
\hline$>1 \mathrm{~cm}$ & $9(7.9)$ & $475.1(151.6-1866.0)$ & $209.6\left(47.4-1500^{*}\right)$ & $39.2(25.9-49.2)$ & $5.46(3.42-7.95)$ & $15.3(8.9-20.3)$ & $5.0(0.8-11.0)$ \\
\hline$P$ value & & 0.034 & 0.238 & 0.056 & 0.028 & $<0.001$ & 0.009 \\
\hline \multicolumn{8}{|l|}{ Endometriosis } \\
\hline Present & $33(28.9)$ & $121.0\left(22.2-5000^{*}\right)$ & $99.5\left(44.1-1500^{*}\right)$ & $41.6(35.7-52.1)$ & $3.83(2.20-7.15)$ & $8.4(5.0-20.0)$ & $1.3(0.2-42.6)$ \\
\hline Absent & $81(70.1)$ & $195.0(6.5-5000)$ & $106.2\left(37.0-1500^{*}\right)$ & $40.7(25.9-49.9)$ & $4.72(1.94-8.94)$ & $11.5(5.0-25.0)$ & $2.1(0.1-55.2)$ \\
\hline$P$ value & & 0.314 & 0.111 & 0.090 & 0.022 & 0.010 & 0.593 \\
\hline \multicolumn{8}{|c|}{ Platinum response } \\
\hline Sensitive & $85(74.6)$ & $105.5\left(6.5-5000^{*}\right)$ & $106.2\left(37.0-1500^{*}\right)$ & $41.7(28.1-52.1)$ & $4.00(1.94-8.94)$ & $9.2(4.9-25.1)$ & $1.0(0.1-30.6)$ \\
\hline resistant & $29(25.4)$ & $378.2(69.93-1866.0)$ & $134.3\left(47.0-1500^{*}\right)$ & $39.2(25.9-49.2)$ & $4.99(2.71-8.79)$ & $13.3(6.2-23.3)$ & $5.0(0.6-55.2)$ \\
\hline$P$ value & & 0.002 & 0.028 & 0.005 & 0.002 & 0.001 & $<0.001$ \\
\hline
\end{tabular}

Abbreviations: $A L B$ albumin, FIB fibrinogen, FAR fibrinogen/ albumin ratio, DDI d-dimer

a Categorical data are shown in absolute value and proportion

b Continuous variables are represented by median and range

* The upper limit of CA125 detection is 5000. The upper limit of HE4 detection is 1500

$P$ values with statistical significance were denoted

and FAR $\geq 12 \%(\mathrm{HR}=5.012, P=0.003)$ were risk factors for platinum resistance in patients with OCCC. In multivariate analysis, FAR $\geq 12 \%(\mathrm{HR}=4.963, P=0.002)$ and $\mathrm{D}$-dimer were independent risk factors for platinum resistance.

\section{Association between FAR and complete cytoreduction in the advanced stage population}

Of the 48 advanced stage patients, $56.3 \%$ had an FAR $\geq 12 \%$. The overall complete resection rate was 47.9 , and $60.9 \%$ in patients with FAR $<12 \%$. As shown in Table 1 , FAR, CA125, D-dimer and FIB were associated with residual tumors. In the advanced-stage population, an FAR $\geq 12 \%(\mathrm{HR}=4.000, P=0.025)$ was further validated as an independent prognostic factor for failure to achieve complete resection after multivariate analysis (Table 3).

\section{Association between FAR and survival in patients with OCCC}

In addition to its predictive value for the platinum response, the FAR was also a predictor of recurrence and death within three years. The optimal cutoff point of FAR is still 12\%. The ROC curve is also shown in Fig. 3.

At the end of the follow-up period (May 30, 2021), 44 patients $(41.9 \%)$ had recurrence, and 31 patients (29.5\%) died. The median follow-up time was 52 months (range, 1-164 months). The relationships between preoperative biochemical variables and survival outcomes are presented in Table 4 . A FAR $\geq 12 \%$ was an independent negative factor for both PFS $(\mathrm{HR}=2.228, P=0.009)$ and OS $(\mathrm{HR}=3.606, P<0.001)$, as well as high $\mathrm{D}$-dimer, advanced stage and residual tumor. The median PFS of all patients was 76 months, and the median OS was not achieved. As shown in Fig. 4, the median PFS and OS were 17 and 


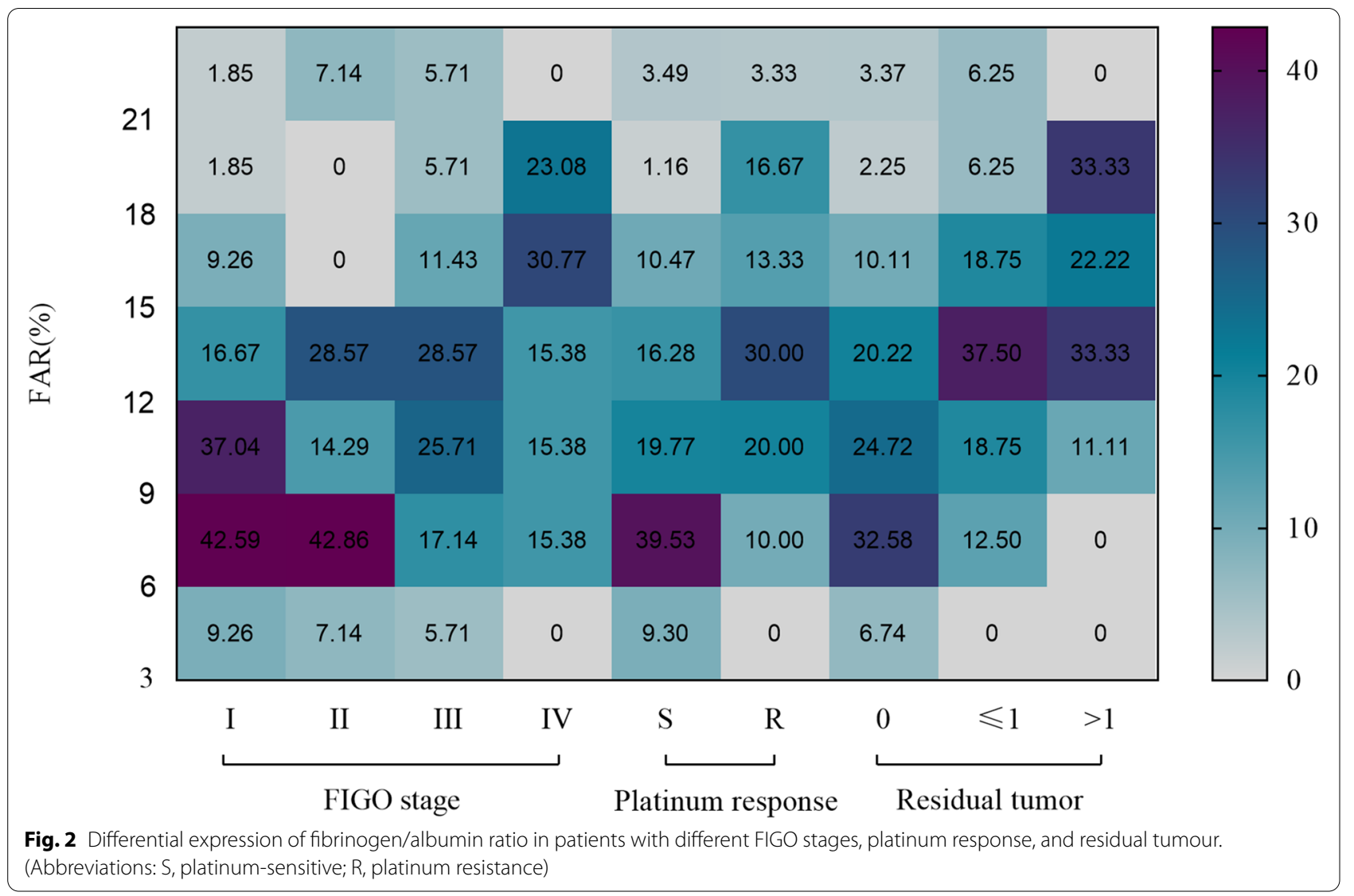

Fig. 2 Differential expression of fibrinogen/albumin ratio in patients with different FIGO stages, platinum response, and residual tumour. (Abbreviations: S, platinum-sensitive; $R$, platinum resistance)

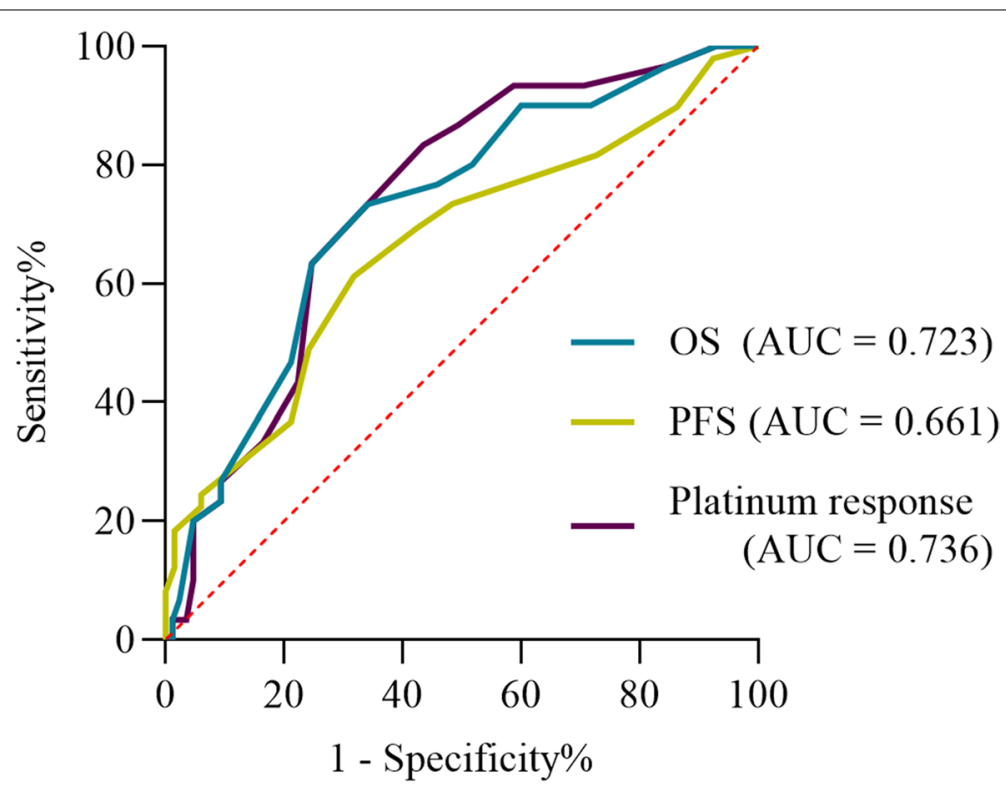

Fig. 3 Receiver operating characteristic curve demonstrating the AUC of preoperative FAR for platinum resistance, PFS and OS. (Abbreviations: AUC, area under the curve; FAR, fibrinogen/albumin ratio; OS, overall survival; PFS, progression-free survival) 
Table 2 Univariate and multivariate analysis regarding platinum response. $(N=114)$

\begin{tabular}{|c|c|c|c|c|c|c|}
\hline \multirow[t]{2}{*}{ Variables } & \multicolumn{3}{|c|}{ Univariate analysis } & \multicolumn{3}{|c|}{ Multivariate analysis } \\
\hline & HR & $95 \% \mathrm{Cl}$ & $P$ & HR & $95 \% \mathrm{Cl}$ & $P$ \\
\hline stage & 2.526 & $1.503-4.248$ & $<0.001$ & & & 0.114 \\
\hline Endometriosis & & & 0.365 & & & \\
\hline CA125 & & & 0.703 & & & \\
\hline HE4 & & & 0.700 & & & \\
\hline ALB & 0.903 & $0.814-1.002$ & 0.055 & & & 0.567 \\
\hline FIB & 1.516 & $1.079-2.130$ & 0.016 & & & 0.661 \\
\hline \multicolumn{7}{|l|}{ FAR(\%) } \\
\hline$<12$ & 1 & & & 1 & & \\
\hline$\geq 12$ & 5.012 & $1.737-14.464$ & 0.003 & 4.963 & $1.845-13.354$ & 0.002 \\
\hline DDI & 1.116 & $1.103-1.23$ & 0.027 & 1.101 & $1.001-1.210$ & 0.048 \\
\hline
\end{tabular}

Abbreviations: $A L B$ albumin, $F I B$ fibrinogen, FAR fibrinogen/ albumin ratio, $D D / d$-dimer, $H R$ hazard ratio, $C I$ confidence interval

$P$ values with statistical significance were denoted

Table 3 Multivariate analysis regarding residual tumour of advanced patients. $(N=48)$

\begin{tabular}{|c|c|c|c|}
\hline \multirow[t]{2}{*}{ Variables } & \multicolumn{3}{|c|}{ Multivariate analysis } \\
\hline & HR & $95 \% \mathrm{Cl}$ & $P$ \\
\hline stage & & & 0.501 \\
\hline Endometriosis & & & 0.124 \\
\hline CA125 & & & 0.765 \\
\hline $\mathrm{FIB}$ & & & 0.250 \\
\hline \multicolumn{4}{|l|}{ FAR (\%) } \\
\hline \multicolumn{4}{|l|}{$<12$} \\
\hline$\geq 12$ & 5.057 & $1.903-13.440$ & 0.001 \\
\hline DDI & & & 0.707 \\
\hline
\end{tabular}

Abbreviations: FIB fibrinogen, FAR fibrinogen/ albumin ratio, $D D I$ d-dimer, $H R$ hazard ratio, $\mathrm{Cl}$ confidence interval

$P$ values with statistical significance were denoted

43 months in the patients with FAR $\geq 12 \%$, respectively. However, median survival was not achieved in patients with FAR $<12 \%$. Survival was significantly worse for the patients with FAR $\geq 12 \%$ than for those with FAR $<12 \%$. Subgroup analyses were conducted based on patients without residual tumor material, advanced stage (FIGO III-IV) and advanced patients who achieved no residual disease. Consequently, a high FAR $(\geq 12 \%)$ showed a stronger correlation with poor OS and PFS across all subgroup analyses (Fig. 5A, B, C).

\section{Discussion}

OCCC is known to be less sensitive to platinum-based front-line chemotherapy and have an overall poor prognosis compared to other types of epithelial ovarian cancers, especially in advanced stages [6]. To date, few clinical and biological biomarkers have been used to predict platinum resistance in OCCC. CA125, fibrinogen, albumin and D-dimer are routine indicators for preoperative examination, making these biomarkers inexpensive and clinically practical. In our previous study, we found that elevated D-dimer and decreased albumin are potential biomarkers for a worse response to front-line platinum chemotherapy and poor clinical outcome in IC-IV stage OCCC patients [18]. However, the combination of D-dimer and albumin did not show better predictive potential for prognosis. The current study extended the patients' enrolment to any stage of OCCC patients who underwent initial surgery. D-dimer was also shown to be significantly associated with prognosis, which is very consistent with findings in other malignancies [11, 19, 20]. D-dimer is the degradation product of fibrin and fibrinogen, and the increase in D-dimer is closely related to fibrinogen. The current study is the first publication evaluating the predictive and prognostic role of the FAR in OCCC. Encouragingly, FAR, as a compound marker of fibrinogen and albumin, has a fairly consistent potential for prognostic evaluation of platinum response and survival outcomes.

In the present study, the patients with an FAR $\geq 12 \%$ had a higher proportion of stage III-IV tumor, platinum resistance and a lower proportion of no residual tumour than the patients with an FAR $<12 \%$. Among advanced ovarian cancers, several studies have reported the best prognosis for patients receiving complete resection [21-23]. Approximately half of OCCC patients were at an advanced stage with widespread intra-abdominal disease in our cohort. FAR showed significant differences between the two groups with or without complete resection. This indicates that OCCC patients with 
Table 4 Multivariate cox proportional analysis regarding overall survival and progression free survival

\begin{tabular}{|c|c|c|c|c|c|c|}
\hline \multirow[t]{2}{*}{ Variables } & \multicolumn{3}{|l|}{ PFS } & \multicolumn{3}{|l|}{ os } \\
\hline & HR & $95 \% \mathrm{Cl}$ & $P$ & HR & $95 \% \mathrm{Cl}$ & $P$ \\
\hline FIB & & & 0.902 & & & 0.922 \\
\hline DDI & 1.045 & $1.013-1.078$ & 0.005 & 1.044 & $1.001-1.089$ & 0.046 \\
\hline CA125 & & & 0.281 & & & 0.143 \\
\hline ALB & & & 0.652 & & & 0.593 \\
\hline HE4 & & & 0.667 & & & 0.587 \\
\hline Endometriosis & & & 0.629 & & & 0.947 \\
\hline \multicolumn{7}{|l|}{ stage } \\
\hline I & 1 & & & 1 & & \\
\hline$\|$ & 3.817 & $1.526-9.551$ & 0.004 & & & 0.951 \\
\hline III & 7.760 & $3.313-18.173$ & $<0.001$ & 8.586 & $3.234-22.796$ & $<0.001$ \\
\hline IV & 3.590 & $1.251-10.308$ & 0.018 & 3.596 & $1.123-11.510$ & 0.031 \\
\hline \multicolumn{7}{|l|}{ Residual tumour } \\
\hline 0 & 1 & & & & & \\
\hline$<=1 \mathrm{~cm}$ & & & 0.410 & & & 0.669 \\
\hline$>1 \mathrm{~cm}$ & 5.556 & 2.079-14.843 & 0.001 & 2.484 & $1.020-6.050$ & 0.045 \\
\hline \multicolumn{7}{|l|}{ FAR } \\
\hline$<12 \%$ & 1 & & & & & \\
\hline$>=12 \%$ & 2.228 & $1.217-4.081$ & 0.009 & 3.606 & $1.759-7.390$ & $<0.001$ \\
\hline
\end{tabular}

Abbreviations: $A L B$ albumin, FIB fibrinogen, FAR fibrinogen/ albumin ratio, $D D I$ d-dimer, $H R$ hazard ratio, $C l$ confidence interval

$P$ values with statistical significance were denoted

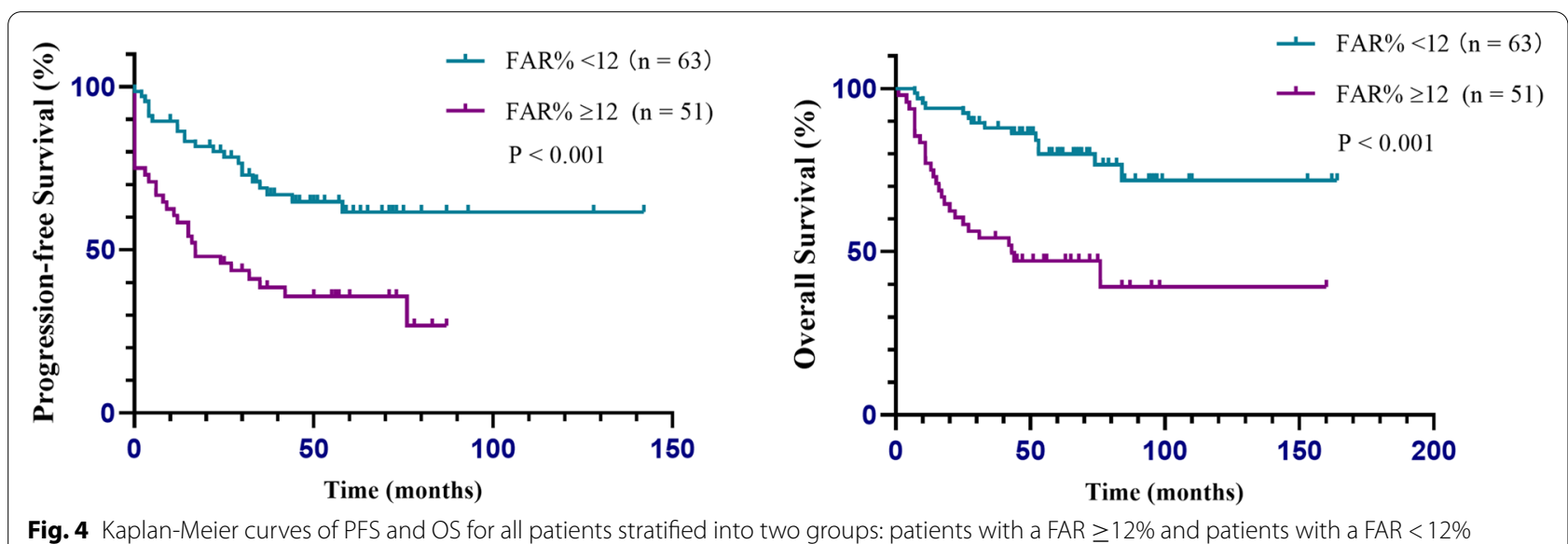

a relatively high FAR may have a high tumor burden and higher tumor invasiveness and may have more difficulty achieving complete resection. An FAR $\geq 12 \%$ is expected to be a complement to predictive models of noninvasive methods.

After a median follow-up of 52 months, the patients with an FAR $\geq 12 \%$ exhibited worse survival than the patients with an FAR $<12 \%$. For the same tumor stage or residual tumor, FAR $\geq 12 \%$ was closely associated with poorer OS and PFS. The results suggested that a preoperative elevated FAR was a useful predictor of a higher risk of recurrence and poor survival in patients with OCCC.

Albumin has been considered an indicator that could not only reflect nutritional status but also be involved in the evaluation system related to the inflammatory response [24]. Hypoalbuminemia can cause immune deficiency in tumor patients, reduce 


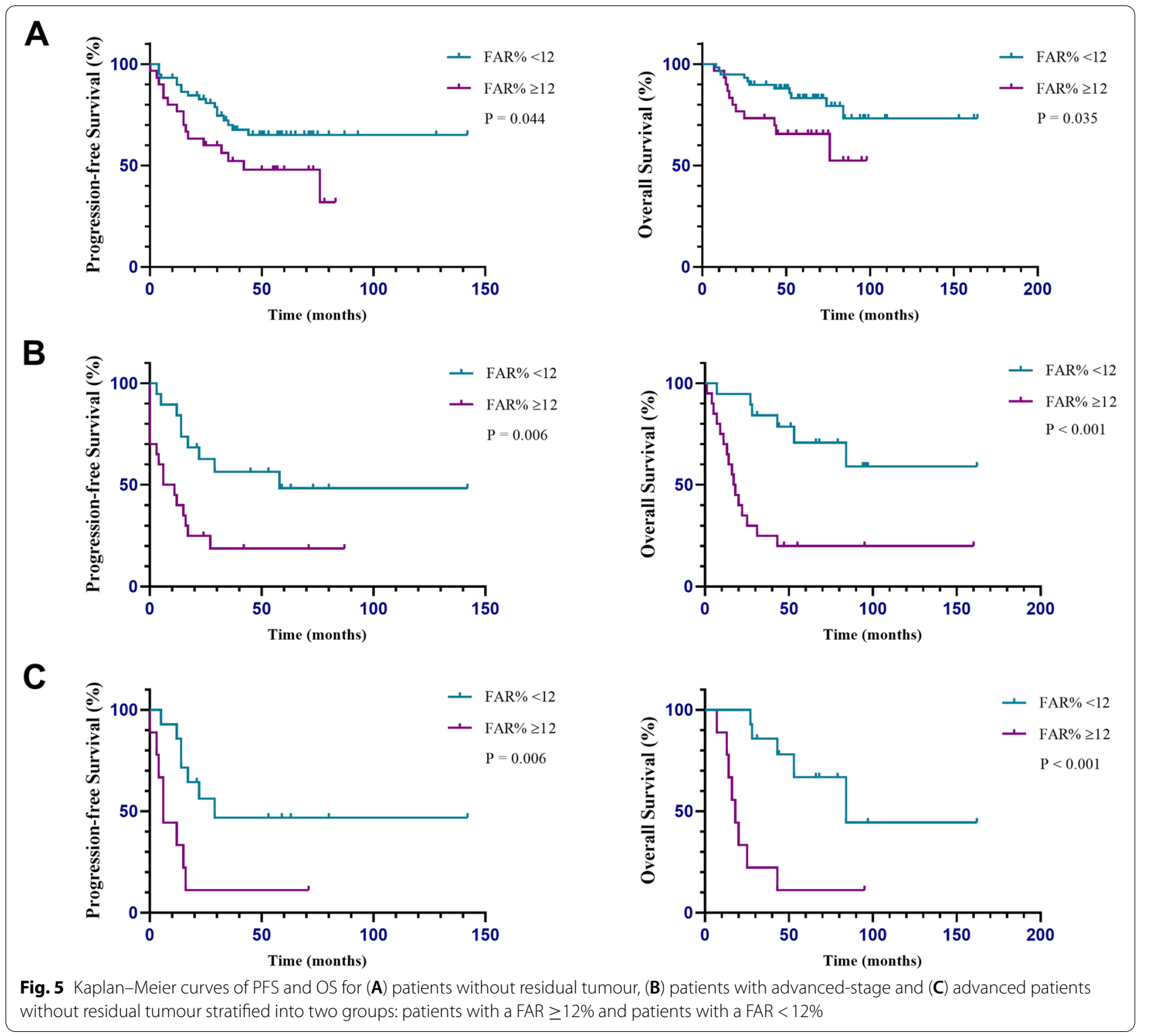

the therapeutic effect and increase mortality [25, 26]. Many observations have shown that fibrinogen, fibrin, and components of the fibrinolytic system have a complex interaction with cancer growth and metastasis [10]. In simple terms, plasma hyperfibrinogen promotes the hypercoagulable state, as well as the adhesion and survival of tumor cells, leading to the metastatic potential of cancer [27]. Fibrinogen can provide a stable framework for the tumor extracellular matrix to promote the adhesion and invasion of tumor cells [28], and block the killing function of natural killer cells to increase the tumor metastasis [29]. In addition, increased levels of circulating fibrinogen can induce the synthesis of interleukin 6 (IL-6) and promote tumor progression [30]. It is known that many OCCC occur in the context of endometriosis. There is evidence that IL-6 expression may be induced by endometriosis inflammation, and long-term exposure of ovarian surface epithelial cells to IL-6-rich endometrioma fluid can induce the expression of gene expression patterns typical of OCCC [31]. This may be one of the pathogeneses of OCCC associated with endometriosis. However, no correlation between endometriosis and the survival of OCCC patients was found in our study, which is consistent with our previous study [32]. In any case, 
FAR, as a composite index of fibrinogen and albumin, not only represents the inflammatory status but also reflects the nutritional status of the body and plays an important role in the biological behavior related to OCCC through direct and indirect pathways.

This study has several limitations. First, our study is a single-center retrospective study with a relatively small sample size, which might lead to selection bias. Second, some confounding factors may not have been eliminated. Third, the median survival time could not be calculated due to insufficient follow-up time. Therefore, the role of the FAR in predicting the clinical response to platinum-based chemotherapy and the survival of OCCC patients needs to be confirmed by a prospective cohort study with a multicenter design and a large sample size.

\section{Conclusion}

We identified the FAR as an independent predictor of platinum response and survival in OCCC patients after primary surgery. The FAR is a potential prognostic indicator of OCCC due to its easy access and low cost.

\section{Abbreviations}

OCCC: Ovarian clear cell carcinoma; EOC: Epithelial ovarian cancer; FAR Fibrinogen/albumin ratio; PFS: Progression-free survival; OS: Overall survival; ROC: Receiver operating characteristic; HR: Hazard ratio; MPFS: median PFS; mOS: median OS; IL-6: interleukin 6.

\section{Acknowledgements}

None.

\section{Authors' contributions}

Conception and design: WC, BS, SL, HY, SY; Collection and assembly of data: WC, BS, SY; Data analysis and interpretation: BS, SL, HY, SY; Manuscript writing: WC, HY, SY; Final approval of manuscript: WC, BS, SL, HY, SY.

\section{Funding}

The study was supported by grants from National Natural Science Foundation of China (81702558) and Shanghai Natural Science Foundation (20ZR1413000). The funding bodies didn't participate in the design of the study and collection, analysis, and interpretation of data and in writing the manuscript.

\section{Availability of data and materials}

The dataset supporting the conclusions of this article is available upon request. Please contact Prof. Shuang Ye (mendy_ye@126.com) and Prof. Huijuan Yang (huijuanyang@hotmail.com).

\section{Declarations}

\section{Ethics approval and consent to participate}

This study was approved by the institutional review board at Fudan University Shanghai Cancer Center. The written informed consent was waived due to retrospective design. All methods were carried out in accordance with relevant guidelines and regulations.

\section{Consent for publication}

Not applicable.

\section{Competing interests}

All the authors have nothing to declare.

\section{Author details}

${ }^{1}$ Department of Gynecologic Oncology, Fudan University Shanghai Cancer Center, Shanghai, China. ${ }^{2}$ Department of Obstetrics and Gynecology, Minhang Hospital, Fudan University, the Central Hospital of Minhang District, Shanghai, China. ${ }^{3}$ Department of Oncology, Shanghai Medical College, Fudan University, Shanghai 200032, China. ${ }^{4}$ Department of Pathology, Fudan University Shanghai Cancer Center, Shanghai, China.

Received: 24 October 2021 Accepted: 17 January 2022

Published online: 21 January 2022

\section{References}

1. Siegel RL, Miller KD, Jemal A. Cancer statistics, 2018. CA Cancer J Clin. 2018;68(1):7-30.

2. Glasspool RM, McNeish IA. Clear cell carcinoma of ovary and uterus. Curr Oncol Rep. 2013;15(6):566-72.

3. Lheureux S, Braunstein M, Oza AM. Epithelial ovarian cancer: evolution of management in the era of precision medicine. CA Cancer J Clin. 2019:69(4):280-304

4. del Carmen MG, Birrer M, Schorge JO. Clear cell carcinoma of the ovary: a review of the literature. Gynecol Oncol. 2012;126(3):481-90.

5. Anglesio MS, Carey MS, Köbel M, Mackay H, Huntsman DG. Clear cell carcinoma of the ovary: a report from the first ovarian clear cell symposium, June 24th, 2010. Gynecol Oncol. 2011;121(2):407-15.

6. Marks El, Brown VS, Dizon DS. Genomic and molecular abnormalities in gynecologic clear cell carcinoma. Am J Clin Oncol. 2020;43(2):139-45.

7. Caine GJ, Stonelake PS, Lip GYH, Kehoe ST. The hypercoagulable state of malignancy: pathogenesis and current debate. Neoplasia. 2002;4(6):465-73

8. Uno K, Homma S, Satoh T, Nakanishi K, Abe D, Matsumoto K, et al. Tissue factor expression as a possible determinant of thromboembolism in ovarian cancer. Br J Cancer. 2007;96(2):290-5.

9. Wang YY, Liu ZZ, Xu D, Liu M, Wang K, Xing BC. Fibrinogen-albumin ratio index (FARI): a more promising inflammation-based prognostic marker for patients undergoing hepatectomy for colorectal liver metastases. Ann Surg Oncol. 2019;26(11):3682-92.

10. Kwaan HC, Lindholm PF. Fibrin and fibrinolysis in Cancer. Semin Thromb Hemost. 2019;45(4):413-22.

11. Lin Y, Liu Z, Qiu Y, Zhang J, Wu H, Liang R, et al. Clinical significance of plasma D-dimer and fibrinogen in digestive cancer: a systematic review and meta-analysis. Eur J Surg Oncol. 2018:44(10):1494-503.

12. Matsuda S, Takeuchi H, Kawakubo H, Takemura R, Maeda Y, Hirata Y, et al. Validation study of fibrinogen and albumin score in esophageal Cancer patients who underwent Esophagectomy: multicenter prospective cohort study. Ann Surg Oncol. 2021;28(2):774-84.

13. Tan Z, Zhang M, Han Q, Wen J, Luo K, Lin P, et al. A novel blood tool of cancer prognosis in esophageal squamous cell carcinoma: the fibrinogen/albumin ratio. J Cancer. 2017;8(6):1025-9.

14. Liang Y, Wang W, Que Y, Guan Y, Xiao W, Fang C, et al. Prognostic value of the fibrinogen/albumin ratio (FAR) in patients with operable soft tissue sarcoma. BMC Cancer. 2018;18(1):942.

15. Xu Q, Yan Y, Gu S, Mao K, Zhang J, Huang P, et al. A novel inflammationbased prognostic score: the fibrinogen/albumin ratio predicts prognoses of patients after curative resection for hepatocellular carcinoma. J Immunol Res. 2018;2018:4925498

16. Zou Y, Zhu Z, Zhou J, Wu X, Li H, Ning X, et al. Fibrinogen/Albumin ratio: A more powerful prognostic index for patients with end-stage renal disease. Eur J Clin Investig. 2020:e13266.

17. Deng S, Fan Z, Xia H, Gong Y, Qian Y, Huang Q, et al. Fibrinogen/albumin ratio as a promising marker for predicting survival in pancreatic neuroendocrine neoplasms. Cancer Manag Res. 2021;13:107-15.

18. Chen W, Zhong S, Shan B, Zhou S, Wu X, Yang H, et al. Serum D-dimer albumin and systemic inflammatory response markers in ovarian clear cell carcinoma and their prognostic implications. J Ovarian Res. 2020:13(1):89. 
19. Hara K, Aoyama T, Hayashi T, Nakazono M, Nagasawa S, Shimoda Y, et al. Postoperative D-dimer elevation affects tumor recurrence and the longterm survival in gastric cancer patients who undergo gastrectomy. Int J Clin Oncol. 2020;25(4):584-94.

20. Watanabe A, Araki K, Harimoto N, Kubo N, Igarashi T, Ishii N, et al. D-dimer predicts postoperative recurrence and prognosis in patients with liver metastasis of colorectal cancer. Int J Clin Oncol. 2018;23(4):689-97.

21. Chang S-J, Bristow RE, Ryu H-S. Impact of complete cytoreduction leaving no gross residual disease associated with radical cytoreductive surgical procedures on survival in advanced ovarian cancer. Ann Surg Oncol. 2012;19(13):4059-67.

22. Aletti GD, Dowdy SC, Gostout BS, Jones MB, Stanhope CR, Wilson TO, et al. Aggressive surgical effort and improved survival in advanced-stage ovarian cancer. Obstet Gynecol. 2006;107(1):77-85.

23. Chi DS, Eisenhauer EL, Lang J, Huh J, Haddad L, Abu-Rustum NR, et al. What is the optimal goal of primary cytoreductive surgery for bulky stage IIIC epithelial ovarian carcinoma (EOC)? Gynecol Oncol. 2006;103(2):559-64.

24. Dupré A, Malik HZ. Inflammation and cancer: what a surgical oncologist should know. Eur J Surg Oncol. 2018;44(5):566-70.

25. Cham S, Chen L, St Clair CM, Hou JY, Tergas Al, Melamed A, et al. Development and validation of a risk-calculator for adverse perioperative outcomes for women with ovarian cancer. Am J Obstet Gynecol. 2019;220(6):571.e571-8.

26. Jansson H, Cornillet M, Björkström NK, Sturesson C, Sparrelid E. Prognostic value of preoperative inflammatory markers in resectable biliary tract cancer - validation and comparison of the Glasgow prognostic score and modified Glasgow prognostic score in a Western cohort. Eur J Surg Oncol. 2020;46(5):804-10.

27. Palumbo JS, Kombrinck KW, Drew AF, Grimes TS, Kiser JH, Degen JL, et al. Fibrinogen is an important determinant of the metastatic potential of circulating tumor cells. Blood. 2000;96(10):3302-9.

28. Sahni A, Simpson-Haidaris PJ, Sahni SK, Vaday GG, Francis CW. Fibrinogen synthesized by cancer cells augments the proliferative effect of fibroblast growth factor-2 (FGF-2). J Thromb Haemost. 2008;6(1):176-83.

29. Palumbo JS, Talmage KE, Massari JV, La Jeunesse CM, Flick MJ, Kombrinck $\mathrm{KW}$, et al. Platelets and fibrin(ogen) increase metastatic potential by impeding natural killer cell-mediated elimination of tumor cells. Blood. 2005;105(1):178-85.

30. Ridker PM, Howard CP, Walter V, Everett B, Libby P, Hensen J, et al. Effects of interleukin-1 $\beta$ inhibition with canakinumab on hemoglobin A1c, lipids, C-reactive protein, interleukin-6, and fibrinogen: a phase llb randomized, placebo-controlled trial. Circulation. 2012;126(23):2739-48.

31. Yamaguchi K, Mandai M, Oura T, Matsumura N, Hamanishi J, Baba T, et al. Identification of an ovarian clear cell carcinoma gene signature that reflects inherent disease biology and the carcinogenic processes. Oncogene. 2010;29(12):1741-52.

32. Ye S, Yang J, You Y, Cao D, Bai H, Lang J, et al. Comparative study of ovarian clear cell carcinoma with and without endometriosis in People's Republic of China. Fertil Steril. 2014;102(6):1656-62.

\section{Publisher's Note}

Springer Nature remains neutral with regard to jurisdictional claims in published maps and institutional affiliations.

Ready to submit your research? Choose BMC and benefit from:

- fast, convenient online submission

- thorough peer review by experienced researchers in your field

- rapid publication on acceptance

- support for research data, including large and complex data types

- gold Open Access which fosters wider collaboration and increased citations

- maximum visibility for your research: over $100 \mathrm{M}$ website views per year

At BMC, research is always in progress.

Learn more biomedcentral.com/submissions 